\title{
Long-Term Cost-Effectiveness Analyses of Empagliflozin Versus Oral Semaglutide, in Addition to Metformin, for the Treatment of Type 2 Diabetes in the UK
}

\author{
Mafalda Ramos - Michael H. Cummings - Anastasia Ustyugova • \\ Syed I. Raza · Shamika U. de Silva · Mark Lamotte
}

Received: June 5, 2020 / Published online: July 22, 2020

(C) The Author(s) 2020

\begin{abstract}
Introduction: International guidelines recommend treatment with a sodium-glucose cotransporter-2 (SGLT-2) inhibitor or glucagonlike peptide-1 (GLP-1) receptor agonist for treatment intensification in type 2 diabetes mellitus (T2DM) patients with progression on metformin. In the randomised, controlled,
\end{abstract}

Digital Features To view digital features for this article go to https://doi.org/10.6084/m9.figshare.12630701.

Electronic supplementary material The online version of this article (https://doi.org/10.1007/s13300020-00883-1) contains supplementary material, which is available to authorized users.

\section{Ramos}

Global HEOR/Real World Solutions, IQVIA, 2740-266 Porto Salvo, Portugal

M. H. Cummings

Academic Department of Diabetes and Endocrinology, Queen Alexandra Hospital, Portsmouth PO6 3LY, Hampshire, UK

\section{A. Ustyugova}

Boehringer Ingelheim International $\mathrm{GmbH}$, 55216 Ingelheim Am Rhein, Germany

\section{S. I. Raza · S. U. de Silva}

Boehringer Ingelheim Ltd., Bracknell RG12 8YS, Berkshire, UK

\section{Lamotte $(\bowtie)$}

Global HEOR/Real World Solutions, IQVIA, 1930 Zaventem, Belgium

e-mail: Mark.lamotte@iqvia.com
Peptide Innovation for Early Diabetes Treatment (PIONEER) 2 trial, the SGLT-2 inhibitor empagliflozin was compared with the GLP-1 receptor agonist oral semaglutide, in addition to metformin. The aim of the current study was to assess the long-term cost-effectiveness of empagliflozin $25 \mathrm{mg}$ versus oral semaglutide $14 \mathrm{mg}$, in addition to metformin, for T2DM patients in the UK.

Methods: Analyses were conducted from the UK healthcare payer perspective, using the IQVIA Core Diabetes model, with a time horizon of 50 years. Patients received either empagliflozin or oral semaglutide, in addition to metformin, until Hba1c threshold of 7.5\% $(58 \mathrm{mmol} / \mathrm{mol})$ was exceeded, following which treatment intensification with insulin glargine in addition to empagliflozin or oral semaglutide plus metformin was assumed. Baseline cohort characteristics and 52-week treatment effects were derived from the PIONEER 2 trial. Treatment effects of empagliflozin and GLP-1 receptor agonists on hospitalisation for heart failure (hHF) were based on the Empagliflozin Comparative Effectiveness and Safety (EMPRISE) real-world study. Utilities, treatment costs and costs of diabetes-related complications were obtained from published sources.

Results: Direct costs for empagliflozin plus metformin were considerably lower than those for oral semaglutide plus metformin (by more than GBP 6000). Compared with oral semaglutide plus metformin, empagliflozin plus 
metformin was a cost-effective treatment for T2DM patients in all scenarios tested. Probabilistic sensitivity analysis showed cost-effectiveness in $>95 \%$ of the iterations using a threshold of 20,000 GBP/QALY.

Conclusion: Empagliflozin $25 \mathrm{mg}$ is a cost-effective treatment option versus oral semaglutide $14 \mathrm{mg}$, when used in addition to metformin, for the treatment of T2DM patients in the UK.

Keywords: Costs and cost analysis; Costeffectiveness; Diabetes mellitus; Empagliflozin; GLP-1 receptor agonists; Oral semaglutide; SGLT-2 inhibitors; Treatment intensification; United Kingdom

\section{Key Summary Points}

\section{Why carry out this study?}

Clinical guidelines recommend treatment with a sodium-glucose cotransporter-2 (SGLT-2) inhibitor or a glucagon-like peptide-1 (GLP-1) receptor agonist in type 2 diabetes mellitus (T2DM) patients with established cardiovascular disease.

As treatment with SGLT-2 inhibitors or GLP-1 receptor agonists, in addition to metformin, are to be continued lifelong, it is important to understand the long-term cost-effectiveness of these therapies for T2DM treatment.

An analysis of the long-term costeffectiveness of treatment with empagliflozin $25 \mathrm{mg}$ versus oral semaglutide $14 \mathrm{mg}$, in addition to metformin, was performed for T2DM patients in the UK setting, including those with hospitalisation for heart failure (hhF).

\section{What was learned from the study?}

Empagliflozin $25 \mathrm{mg}$ is a cost-effective treatment option versus oral semaglutide $14 \mathrm{mg}$, when used in addition to metformin, for the treatment of T2DM patients in the UK.
Empagliflozin plus metformin was dominant (less costly and generated more health gains) to oral semaglutide plus metformin in the treatment of T2DM patients with hHF.

\section{INTRODUCTION}

Management of type 2 diabetes mellitus (T2DM) is associated with considerable burden to the health care system in the UK, estimated to cost $£ 10$ billion each year [1]. Hypoglycaemic episodes, diabetes-related complications and comorbidities contribute to reduced health-related quality of life (HRQL) and increased health care resource utilisation and costs in patients with T2DM [2]. Inadequate glycaemic control is associated with the development of macrovascular complications (coronary ischaemia, stroke, myocardial infarction [MI] and angina pectoris) [3] and microvascular complications (e.g. retinopathy, nephropathy and neuropathy) [2]. T2DM patients have a two- to four-fold increased risk of cardiovascular disease (CVD) compared with a nondiabetic population, and more than half die from CVD complications $[4,5]$. By reducing adverse outcomes, effective diabetes management providing even modest improvements in glycaemic control can achieve considerable cost savings [3, 6].

Since 2008, based on US Food and Drug Administration recommendations, any new T2DM therapy must demonstrate that it is not associated with an unacceptable increase in cardiovascular risk, which has led to several cardiovascular outcomes trials (CVOTs) [7]. Many studies have demonstrated that the trial therapy does not result in an unacceptable increase in cardiovascular risk such as major cardiovascular events (MACE) and all-cause mortality [7]. Additionally, treatments with the sodium-glucose cotransporter-2 (SGLT-2) inhibitor class of glucose-lowering agents together with standard of care have shown a significant reduction in MACE in a number of meta-analyses of CVOT trials in patients with T2DM [8-11]. Tang et al. in 2016 [12] conducted a 
meta-analysis including 37 trials and 29,859 patients with T2DM, comparing canagliflozin, dapagliflozin and empagliflozin with placebo and other glucose-lowering treatments. Only empagliflozin was associated with a significantly lower risk of MACE compared with placebo [odds ratio (OR): 0.81; 95\% CI: 0.70-0.93] [12]. Real-world data from the EMPRISE (Empagliflozin Comparative Effectiveness and Safety) study [13] has shown that empagliflozin is associated with reduced hospitalisation for heart failure (hHF) compared with dipeptidyl peptidase 4 (DPP-4) and glucagon-like peptide-1 (GLP-1) receptor agonists.

The aim of T2DM treatment is to achieve glycaemic control according to individualised haemoglobin $\mathrm{A} 1 \mathrm{C}\left(\mathrm{HbA}_{1 \mathrm{c}}\right)$ targets, initially using metformin together with lifestyle interventions. As T2DM is a chronic, progressive condition, most patients ultimately require intensification of treatment to maintain glycaemic control [14]. Timely intensification of treatment is important to avoid exposure of patients to chronic elevations of $\mathrm{HbA}_{1 \mathrm{c}}$ that may increase the risk of diabetes-related complications and long-term healthcare costs $[15,16]$. When glycaemic control is no longer achieved with metformin alone, dual therapy with glucose-lowering agents is recommended taking into consideration the clinical characteristics of the patient, according to the consensus guidelines of the American Diabetes Association (ADA) and the European Association for the Study of Diabetes (EASD) [17]. Established CVD is considered a compelling indication for treatment with an SGLT-2 inhibitor or GLP-1 receptor. The guidelines further state that the decision to treat appropriate highrisk individuals to reduce MACE, hHF, cardiovascular death or chronic kidney disease (CKD) progression should be considered independently of baseline HbA1c or individualised HbA1c target [17]. In the UK, the National Institute for Health and Care Excellence (NICE) recommends SGLT-2 inhibitors such as empagliflozin for the treatment of T2DM, in combination with insulin with or without other glucose-lowering drugs, or in triple regimens in combination with metformin [18]. GLP-1 receptor agonists are recommended for patients who require subsequent treatment intensification and for whom insulin therapy would have significant occupational implications or for whom weight loss would benefit other obesityrelated comorbidities [19].

However, it can be envisaged that NICE recommendations could be adapted in the near future, based on the recent ADA/EASD recommendations. Thus, providing results of a costeffectiveness analyses that compares an SGLT-2 inhibitor and a GLP-1 receptor agonist can be of interest for the payer.

Oral semaglutide is a new formulation of the GLP-1 receptor agonist semaglutide developed for once-daily oral administration [20-22]. The efficacy and safety of oral semaglutide was assessed in the Peptide Innovation for Early Diabetes Treatment (PIONEER) trial programme [23], including a comparison versus once-daily empagliflozin (PIONEER 2 trial) [22]. As there are only few direct clinical comparisons available between a SGLT-2 inhibitor and a GLP-1 receptor agonist, it seemed of interest to use this PIONEER 2 trial as the basis for a cost-effectiveness analysis.

The objective of the current study was to evaluate the long-term cost-effectiveness of timely initiation of treatment with empagliflozin $25 \mathrm{mg}$ versus oral semaglutide $14 \mathrm{mg}$, in addition to metformin, for T2DM patients in the UK. Separate scenarios were modelled incorporating the treatment effect on hHF.

\section{METHODS}

\section{Modelling Approach}

Using the IQVIA Core Diabetes Model (CDM) version 9.5, long-term projections of clinical and cost outcomes were performed from the UK National Health Service perspective. CDM is a proprietary, interactive, internet-based, computer simulation model developed to determine the long-term health outcomes and economic implications of therapeutic interventions for type 1 and type 2 diabetes. The CDM and its validation studies have been previously described [24-26]. 
Table 1 Treatment effects applied in the analysis

\begin{tabular}{llll}
\hline & Empagliflozin $(\mathrm{SE})$ & Oral semaglutide (SE) & Source \\
\hline Change in baseline HbA1c, \% & $-0.90(0.026)$ & $-1.30(0.026)$ & Rodbard et al. 2019 [22] \\
SBP change from baseline, mmHg & $-4.34(0.63)$ & $-4.85(0.65)$ & Bain et al. 2020 [20] \\
DBP change from baseline, mmHg & $-2.67(0.44)$ & $-2.27(0.45)$ & Bain et al. 2020 [20] \\
Total cholesterol change from baseline, $\mathrm{mg} / \mathrm{dl}$ & $4.74(1.57)$ & $-5.08(1.62)$ & Bain et al. 2020 [20] \\
HDL cholesterol change from baseline, $\mathrm{mg} / \mathrm{dl}$ & $3.11(0.34)$ & $0.73(0.35)$ & Bain et al. 2020 [20] \\
BMI change from baseline, $\mathrm{kg} / \mathrm{m}^{2}$ & $-1.294(0.028)$ & $-1.357(0.028)$ & Rodbard et al. 2019 [22] \\
\hline
\end{tabular}

For the base case analysis, results based on the treatment estimand of the PIONEER 2 study were used $B M I$ body mass index, $D B P$ diastolic blood pressure, $H b A 1 c$ haemoglobin A1c, $H D L$ high-density lipoprotein, $S B P$ systolic blood pressure, $S E$ standard error

Table 2 Adverse events applied in the analysis

\begin{tabular}{llll}
\hline & Empagliflozin & Oral semaglutide & Source \\
\hline NSHE rate, per 100 patient-years & 9.535 & 10.976 & Rodbard et al. 2019 [22] \\
SHE1 rate, per 100 patient-years & 0.244 & 0.244 & Rodbard et al. 2019 [22] \\
SHE2 rate, patient-years & 0 & 0 & Rodbard et al. 2019 [22] \\
\hline
\end{tabular}

NSHE non-severe hypoglycaemia rate, SHE1 severe hypoglycaemia rate (not requiring medical assistance), SHE2 severe hypoglycaemia rate (requiring medical assistance)

Projected outcomes include incidence of complications, rates of clinical events, per patient costs, life-years gained and quality-adjusted life-years (QALYs) gained, over a lifelong time horizon (up to 50 years). Cost-effectiveness was described in terms of: the incremental costutility ratio (ICUR), which is the cost per additional unit of QALY gained for the intervention versus the alternative; the net monetary benefit (NMB), which represents the value of an intervention in monetary terms when a willingnessto-pay (WTP) threshold for a unit of benefit is known. Both costs and effects were discounted by $3.5 \%$ annually, in line with the NICE reference case [27].

Two main scenarios were examined in the modelling analyses: treatment benefit on risk factors, e.g. HbA1c, body-mass index (BMI) and blood pressure; potential hHF treatment benefit of empagliflozin.

\section{Clinical Data}

The cost-effectiveness analysis is based on the efficacy results of the PIONEER 2 study, which enrolled T2DM patients with HbA1c values between 7.0 and $10.5 \% \quad(53-91 \mathrm{mmol} / \mathrm{mol})$, who were treated with metformin. Hence, the baseline characteristics incorporated in the model are the reported weighted average of the baseline cohort from the published clinical trial [22] or cost-effectiveness analyses [20]. Baseline CVD and microvascular complications were based on cohort data from other studies that enrolled a similar patient population (Supplementary material Table S1).

The effects of each of the drugs on HbA1c, systolic blood pressure (SBP), diastolic blood pressure (DBP), total cholesterol, HDL-cholesterol and BMI (Table 1), as well as on hypoglycaemia rates (Table 2), were as reported in the PIONEER-2 study $[20,22]$ and were also obtained from the PIONEER-2 study [22]. In the 
PIONEER 2 study, efficacy was assessed in the context of two estimands: treatment policy and trial product. The treatment policy estimand evaluated the treatment effect for all randomised patients, regardless of trial product discontinuation or use of rescue medication (intention-to-treat principle). The trial product estimand evaluated the treatment effect for all randomised patients under the assumption that all patients remained on trial product for the entire planned duration of the trial and did not use rescue medication, thus reflecting the effect of oral semaglutide compared with empagliflozin without the confounding effect of rescue medication [22]. In the current cost-effectiveness analysis, the data from the treatment policy estimand were used for treatment effects. Additional analyses were performed using the data on treatment effects from the trial product estimand (Supplementary material Table S2).

\section{Treatment Intensification and Long-Term Disease Progression}

Disease progression may be observed as a rise in HbA1c while on the same drug regimen, requiring intensification of therapy in order to regain glycaemic control [28]. Patients were assumed to receive either empagliflozin or oral semaglutide, in addition to metformin, until Hba1c of $7.5 \%(58 \mathrm{mmol} / \mathrm{mol})$ was exceeded; this is the threshold for treatment intensification, defined in the NICE guidelines [19]. As soon as this threshold was exceeded, patients were assumed to intensify treatment with insulin glargine in addition to empagliflozin or oral semaglutide plus metformin, which would be continued lifelong in line with the combined ADA/EASD recommendations that SGLT2 and GLP1 receptor agonists are to be administered irrespective of the HbA1c measure [17].

Following the first year of treatment (study duration was 52 weeks), HbA1c and blood pressure were modelled to follow the UKPDS 68 progression equation for the remainder of patient lifetimes. Mortality was calculated using the UKPDS 82 combined mortality approach. The effect on BMI was assumed to be maintained while the patient remained on empagliflozin or oral semaglutide.

\section{Effect on hHF}

In the EMPRISE study [13], empagliflozin was compared with DPP4 inhibitors and GLP1 receptor agonists in terms of hospitalisation for hHF and atherosclerotic cardiovascular events (MI, unstable angina, stroke and coronary revascularisation), using real-world data from Medicare and two commercial insurance claims databases in the US, over a period of 5 years. For empagliflozin, there was a significant reduction in the rate of hospitalisation for hHF, and a favourable (but statistically non-significant) trend in the rate of atherosclerotic cardiovascular events, compared with DPP4 inhibitors and GLP1 receptor agonists [13]. In the base case analysis and exploratory scenario analyses the potential hHF treatment benefit of empagliflozin was considered (Table 1).

\section{Patient Management}

Patient management inputs included the proportion of patients on preventative medication, proportion of patients undergoing routine screening for diabetic complications and the sensitivity and specificity of the screening tests performed, using UK-specific data where available.

\section{Utilities}

Health-state utilities and event disutilities were based on published sources (Supplementary material Table S3).

\section{Costs}

Costs were accounted from a UK healthcare payer perspective. In addition to treatment costs (Supplementary material Table S4), direct costs also included the costs of treating hypoglycaemic events and long-term complications associated with T2DM (Supplementary material Table S5). In terms of the latter, a distinction 
was made between costs arising in the first year after disease onset and subsequent years, as some complications are associated with high initial costs arising from the need for hospitalisation in the acute phase. Follow-up costs were accounted for every year until resolution of the specific complication.

In the modelling analysis, treatment doses of $25 \mathrm{mg}$ for empagliflozin and $14 \mathrm{mg}$ for oral semaglutide were used, as in the PIONEER-2 trial. Metformin was included at a dose of $1500 \mathrm{mg} /$ day in both treatment arms. Costs of empagliflozin, oral semaglutide and metformin were obtained from Bain et al. [20] and crosschecked against the British National Formulary.

For insulin glargine, the cost of biosimilar Abasaglar $(0.24 £ / U)$ was used. Costs of glucose monitoring and injection needles were obtained from [20] (supplementary material) [20]. Reduction in HbA1c was based on an insulin-naive population derived from the "Core" multivariate equations estimated by Willis et al. [29]. For initial treatment intensification, insulin glargine at a dose of $0.7 \mathrm{IU} / \mathrm{kg}$ was assumed, and for further treatment intensification (exploratory analysis), a dose of $0.9 \mathrm{IU} / \mathrm{kg}$ was assumed for an average body weight of $91.6 \mathrm{~kg}$ [29].

\section{Analytical Approach}

The base case analysis examined treatment with empagliflozin or oral semaglutide, in addition to metformin, until Hba1c of $7.5 \%$ was exceeded, following which patients underwent treatment intensification with insulin glargine in addition to empagliflozin or oral semaglutide plus metformin. The value of empagliflozin plus metformin was quantified by calculating the net monetary benefit (NMB). NMB is defined as the change in health outcomes in terms of QALYs $(\Delta E)$ multiplied by the amount the decision maker is willing to pay (WTP) per unit of increased effectiveness, minus the aggregate direct and treatment costs $(\Delta C)$, relative to oral semaglutide plus metformin. Thus, NMB $=$ WTP ${ }^{*} \Delta E-\Delta C$. In the current analyses, a willingnessto-pay/accept threshold of GBP 20,000 per
QALY gained was used, in line with the typical threshold used by NICE [30].

As extrapolation of long-term clinical outcomes is associated with uncertainty, exploratory scenario analyses were conducted to evaluate how changes to key parameters in the modelling analyses influence the results of the base case analyses. In one of the exploratory scenarios, following initial treatment intensification with insulin glargine in addition to empagliflozin or oral semaglutide, patients who subsequently exceeded the HbA1c threshold of 7.5\% again underwent further intensification with a higher dose of insulin glargine alone to achieve glycaemic control. In these patients, it was assumed that upon further treatment intensification and discontinuation of empagliflozin or oral semaglutide, BMI reverted immediately to baseline values. Another exploratory analysis considered the impact of BMI on health-related quality of life (HRQL), selecting the polynomial model within the CDM to determine BMI utilities. This approach uses polynomial equations to calculate HRQL associated with different BMI values; thus, losing one unit of BMI does not have the same effect on HRQL for a baseline BMI of 27 or 37. Two separate polynomial equations were used to account for differences in gender, as described in Soltoft et al. [31]. Other exploratory analyses included: shortening the time horizon of the analyses to 5 years; treatment intensification with insulin glargine plus empagliflozin or oral semaglutide occurring when HbA1c threshold of $8 \%$ is exceeded. All exploratory analyses were conducted for comparisons including both treatment effect on hHF and excluding treatment effect on hHF.

Probabilistic sensitivity analyses were performed to ascertain the uncertainty around cost-effectiveness outcomes, using Monte Carlo simulations together with a non-parametric bootstrapping approach. Treatment effects, complication costs and utilities were sampled from distributions. Progression of diabetes was simulated in cohorts of 1000 patients run through the model 1000 times. 


\section{Compliance with Ethics Guidelines}

This article is based on previously conducted studies and does not contain any studies with human participants or animals performed by any of the authors.

\section{RESULTS}

\section{Primary Scenario Analyses}

In long-term projections of clinical outcomes taking into account the effect of the treatment on hHF, empagliflozin plus metformin was associated with a gain in life-years of 0.086 and a gain in QALYs of 0.023 compared with oral semaglutide plus metformin. Overall costs of treatment with empagliflozin plus metformin were considerably lower than that of oral semaglutide plus metformin (by GBP 6248; Table 3). Assuming a willingness to pay/accept threshold of GBP 20,000 per QALY gained, empagliflozin plus metformin resulted in a NMB of 6708 . Thus, in the base case scenario in which patients underwent treatment intensification with empagliflozin or oral semaglutide upon exceeding HbA1c levels at a threshold of $7.5 \%$, empagliflozin was dominant to oral semaglutide, i.e. empagliflozin is both less costly and results in better health outcomes than oral semaglutide (Table 4).

When the effect of treatment on hHF was not taken into account, empagliflozin plus metformin was associated with decreases in overall costs of GBP 6161 and also resulted in slightly lower life-years gained $(-0.007)$ and QALYs gained (-0.033) compared with oral semaglutide plus metformin. Assuming a willingness to pay/accept threshold of GBP 20,000 per QALY gained, empagliflozin plus metformin resulted in a NMB of GBP 5501. As the NMB is greater than the incremental cost, empagliflozin plus metformin can be considered cost-effective compared with oral semaglutide plus metformin, even when the effect of treatment on hHF is not taken into consideration.

\section{Exploratory Scenario Analyses}

The results of the exploratory scenario analyses are presented in Table 4 . In the first scenario analysis, following initial treatment intensification with insulin glargine plus empagliflozin or oral semaglutide, patients who subsequently exceeded the HbA1c threshold of $7.5 \%$ underwent further intensification with a higher dose of insulin glargine alone. Empagliflozin plus metformin provided additional life-years gain (0.012) but a slightly lower QALY gain $(-0.025)$ and was less costly compared with oral semaglutide plus metformin (-GBP 2094) in the analysis that included the benefit on hHF. Thus, empagliflozin plus metformin can be considered dominant compared with oral semaglutide plus metformin. When the benefit on hHF was not considered, empagliflozin plus metformin was less costly (-GBP 2048) and resulted in a marginally lower life-years gain $(-0.01)$ and QALY gain $(-0.04)$ compared with oral semaglutide plus metformin.

Similar to the base case analysis, when a higher HbA1c threshold of $8 \%$ was applied for treatment intensification (addition of insulin glargine to either empagliflozin or oral semaglutide), the use empagliflozin plus metformin as initial treatment remained dominant, with additional life-years (0.09) and QALYs (0.02) gained, and lower costs (-GBP 6279), compared with oral semaglutide plus metformin. In this analysis, patients on empagliflozin plus metformin underwent treatment intensification after 4 years, while patients on oral semaglutide plus metformin underwent treatment intensification after 5 years. When the effect of treatment on hHF was not considered, empagliflozin plus metformin was associated with marginally lower gains in life-years $(-0.02)$ and QALYs $(-0.05)$, but was also less costly (-GBP 6268) compared with oral semaglutide plus metformin (Table 4).

Use of the polynomial approach to determine utilities associated with BMI led to increased QALYs with both treatments compared with the base case scenario. Empagliflozin plus metformin remained dominant compared with oral semaglutide plus metformin, with a $\mathrm{NMB}$ of 6768 . When the benefit on hHF was not 
considered, a NMB of GBP 5398 was obtained for the use of empagliflozin plus metformin compared with oral semaglutide plus metformin. Thus, empagliflozin plus metformin can be considered cost-effective compared with oral semaglutide plus metformin when using the polynomial approach to determine BMI-associated utilities (Table 4).

Applying a shorter time horizon of 5 years led to a reduction in life-years and QALYs gained with either treatment. Overall costs, including both treatment costs and costs associated with diabetes-related complications, were lower with empagliflozin plus metformin compared with oral semaglutide plus metformin (Table 3). A NMB of GBP 1116 was obtained when the effect on hHF was considered and GBP 1040 when the effect on hHF was not considered. Thus, empagliflozin plus metformin can be considered cost-effective compared with oral semaglutide plus metformin over a 5-year time horizon (Table 4).

\section{Probabilistic Sensitivity Analysis}

Differences in costs and health outcomes between the two treatments resulting from each simulation were presented in a cost-effectiveness plane and cost-effectiveness acceptability curve. In the analysis including the hHF benefit, the results were delivered in the south-west and south-east of the quadrants (Fig. 1a), indicating that empagliflozin plus metformin generates lower costs compared with oral semaglutide plus metformin. Empagliflozin plus metformin was dominant (less costly and generated more health gains) in $61 \%$ of simulations, and the probability of being cost-effective compared with oral semaglutide plus metformin at the defined WTP threshold of GBP 20,000 per QALY gained was $97 \%$ (Fig. 1b). In the probabilistic sensitivity analysis that did not consider the treatment effect on hHF, the results were also delivered in the south-west and south-east of the quadrants (Fig. 1c). Empagliflozin plus metformin was dominant in $44 \%$ of simulations, and the probability of being cost-effective compared with oral semaglutide plus metformin at the defined WTP threshold of GBP 20,000 per QALY gained was 95\% (Fig. 1d).

\section{DISCUSSION}

Empagliflozin plus metformin is a cost-effective treatment option versus oral semaglutide plus metformin for patients with T2DM requiring treatment intensification. Treatment costs were considerably lower with empagliflozin plus metformin than with oral semaglutide, with clinical benefits also observed with the former in terms of reduced incidence of hHF. Therefore, in patients undergoing treatment intensification, empagliflozin plus metformin was dominant to oral semaglutide plus metformin at a WTP threshold of GBP 20,000. Even without the inclusion of treatment effect on hHF, a positive NMB was observed for empagliflozin plus metformin compared with oral semaglutide plus metformin, with treatment costs being the key driver of cost-effectiveness. The exploratory analyses supported the results of the base case analyses, demonstrating the costeffectiveness of empagliflozin plus metformin compared with oral semaglutide plus metformin from the UK healthcare perspective, in each of the scenarios examined.

A separate cost-effectiveness analysis was recently published [20], comparing oral semaglutide and empagliflozin, which projected that oral semaglutide would be a cost-effective treatment option versus empagliflozin. Similar to the current study, the modelling analysis by Bain et al. [20] was based on the results of the PIONEER 2 clinical trial, which did not include cardiovascular outcomes. The current study additionally utilises real-world data on hHF from the EMPRISE study to separately consider the clinical benefits of empagliflozin on hHF in the base case analysis and each of the exploratory analyses. SGLT-2 inhibitors with evidence of reducing hHF are recommended in T2DM patients with hHF [17]; therefore, this is an additional important consideration assessed in the current cost-effectiveness study.

As EMPRISE was a real-world evidence study, the data provided are for GLP-1 receptor agonists that were currently available for clinical 

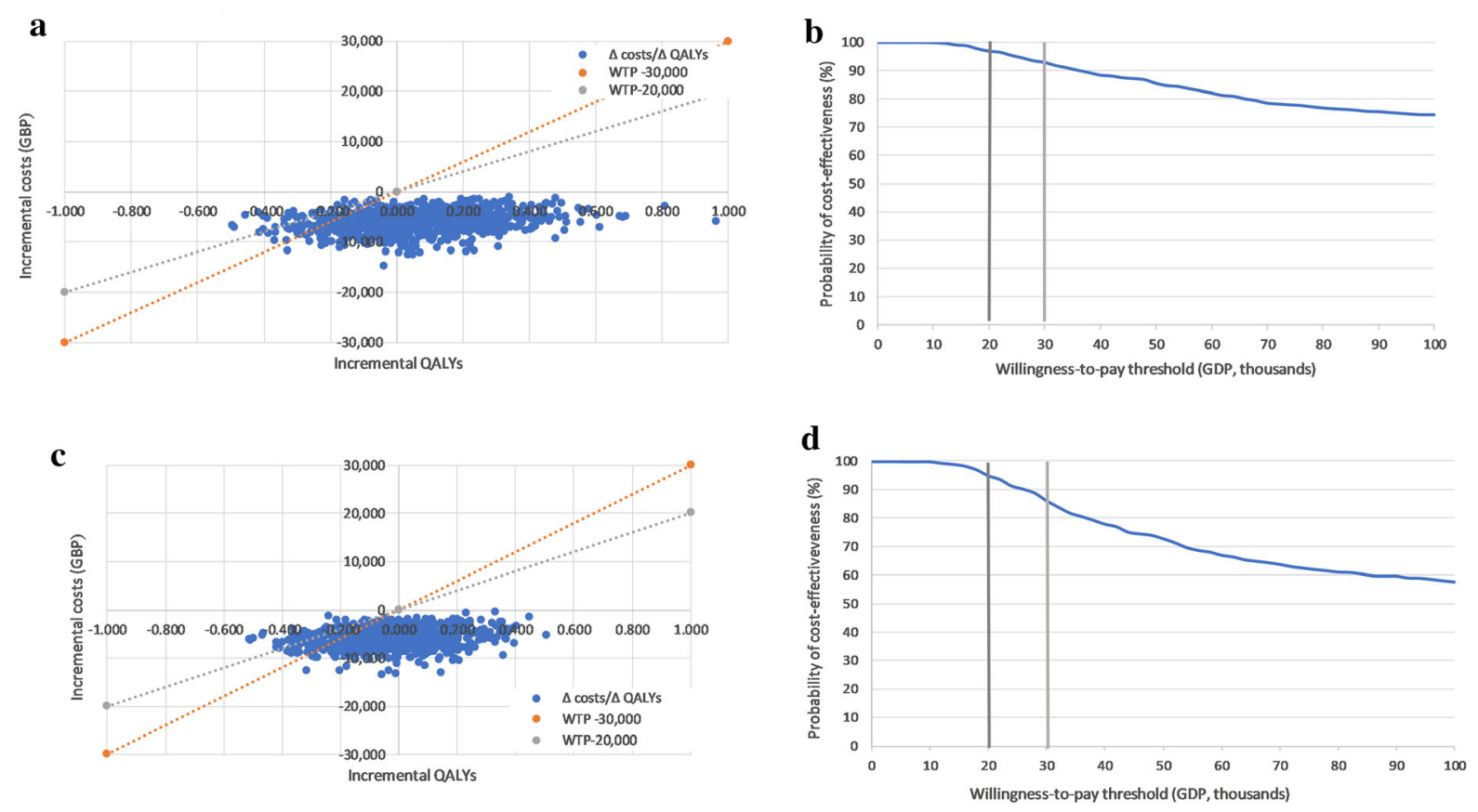

Fig. 1 Cost-effectiveness scatterplots and acceptability curves based on the probabilistic sensitivity analyses. a Cost-effectiveness plane of the base case analysis with hHF benefit (QALY). b Cost-effectiveness acceptability curve of the base case analysis with hHF benefit (QALY). c Cost-effectiveness plane of the base case analysis without

hHF benefit (QALY). d Cost-effectiveness acceptability curve of the base case analysis without hHF benefit (QALY). GBP Pound sterling, $h H F$ hospitalisation for heart failure, $Q A L Y$ quality-adjusted life year, WTP willingness to pay

use and therefore did not include oral semaglutide. Thus, a limitation of the current study is the use of data describing a class effect of GLP-1 receptor agonists rather than the specific effect of oral semaglutide on hHF in the scenarios that consider this treatment effect. However, as the results have shown, even when the impact on hHF was not considered, empagliflozin plus metformin remained cost-effective compared with oral semaglutide plus metformin.

In the PIONEER 2 trial, two sets of data analyses were reported: the trial product estimand (on trial product and without the use of rescue medication) and the treatment policy estimand (which represents an intention-totreat approach) [22]. The cost-effectiveness analyses by Bain et al. [20] were based on data from the trial product estimand, whereas the treatment policy estimand was chosen for the current cost-effectiveness analyses, as this is

more reflective of the real-world situation by not excluding discontinuation of the drug or rescue medication. However, it should be noted that the use of rescue medication and other diabetes medication was not significantly different between the two arms [22]. In additional modelling analyses performed using the trial product estimand, empagliflozin plus metformin remained cost-effective compared with oral semaglutide plus metformin (Supplementary material Table S6).

Based on the NICE algorithm for blood glucose-lowering therapy in T2DM patients [32], those receiving dual therapy with metformin and SGLT-2 (or another oral agent) would undergo treatment intensification when HbA1c threshold of $7.5 \%$ is exceeded. In the modelling analysis conducted by Bain et al. [20], treatment with empagliflozin or oral semaglutide, in addition to metformin, was ceased when HbA1c threshold of $7.5 \%$ was exceeded, assumed to 
Table 3 Direct medical costs (base case analysis)

\begin{tabular}{lccc}
\hline & $\begin{array}{l}\text { Including hHF impact } \\
\text { Empagliflozin + metformin }\end{array}$ & Excluding hHF impact & \\
\cline { 3 - 4 } & & Empagliflozin + metformin & $\begin{array}{l}\text { Oral } \\
\text { semaglutide + metformin }\end{array}$ \\
\hline Total cost (GBP) & 28,193 & 28,280 & 34,441 \\
Treatment & 15,979 & 15,888 & 22,192 \\
Management & 731 & 727 & 727 \\
CVD & 6785 & 6954 & 6960 \\
Renal & 873 & 924 & 896 \\
Ulcer/amputation/ & 945 & 935 & 911 \\
$\quad$ neuropathy & & & 2310 \\
Eye & 2406 & 2381 & 264 \\
Non-severe hypoglycaemia & 284 & 282 & 135 \\
$\begin{array}{l}\text { Severe hypoglycaemia } \\
\text { (required non-medical }\end{array}$ & 140 & 140 & \\
assistance) & & & \\
$\begin{array}{l}\text { Severe hypoglycaemia } \\
\text { (required medical }\end{array}$ & 51 & 50 & \\
assistance) & & & \\
\hline
\end{tabular}

Costs presented are for empagliflozin or oral semaglutide in addition to metformin as dual therapy $C V D$ cardiovascular disease, GBP Pound sterling, $h H F$ hospitalisation for heart failure

occur after 2 years of treatment with empagliflozin and after 3 years of treatment with oral semaglutide, and patients were switched to treatment with insulin glargine alone. Thus, in the Bain et al. study, [20] the treatment effects and costs of empagliflozin or oral semaglutide consider a short duration of 2-3 years of treatment only. This is a key difference from the current modelling analysis, in which treatment with empagliflozin or oral semaglutide, in addition to metformin, was continued lifelong, with insulin glargine added to the drug regimen for treatment intensification when $\mathrm{HbA1c}$ threshold of $7.5 \%$ was exceeded. The latter approach is in line with the combined ADA/ EASD recommendations that treatments with SGLT2 and GLP1 receptor agonists are to be continued irrespective of the HbA1c measure [17].
Based on NICE guidance [NG28] [19], treatment intensification involves triple therapy with metformin in combination with other oral agents, or insulin-based treatment. Combination therapy with metformin and a GLP-1 receptor agonist would be considered only in those patients who have a BMI of $35 \mathrm{~kg} / \mathrm{m}^{2}$ or higher and specific psychological or other medical problems associated with obesity, or for whom insulin therapy would have significant occupational implications, or weight loss would benefit other significant obesity-related comorbidities [32]. There is little clinical evidence on the individual effects of oral agents when used in triple therapy regimens. Hence, modelling of triple oral therapy regimens would be based purely on assumptions regarding efficacy, safety and occurrence of diabetes-related complications. Therefore, as in the current study, other studies modelling T2DM treatment regimens 


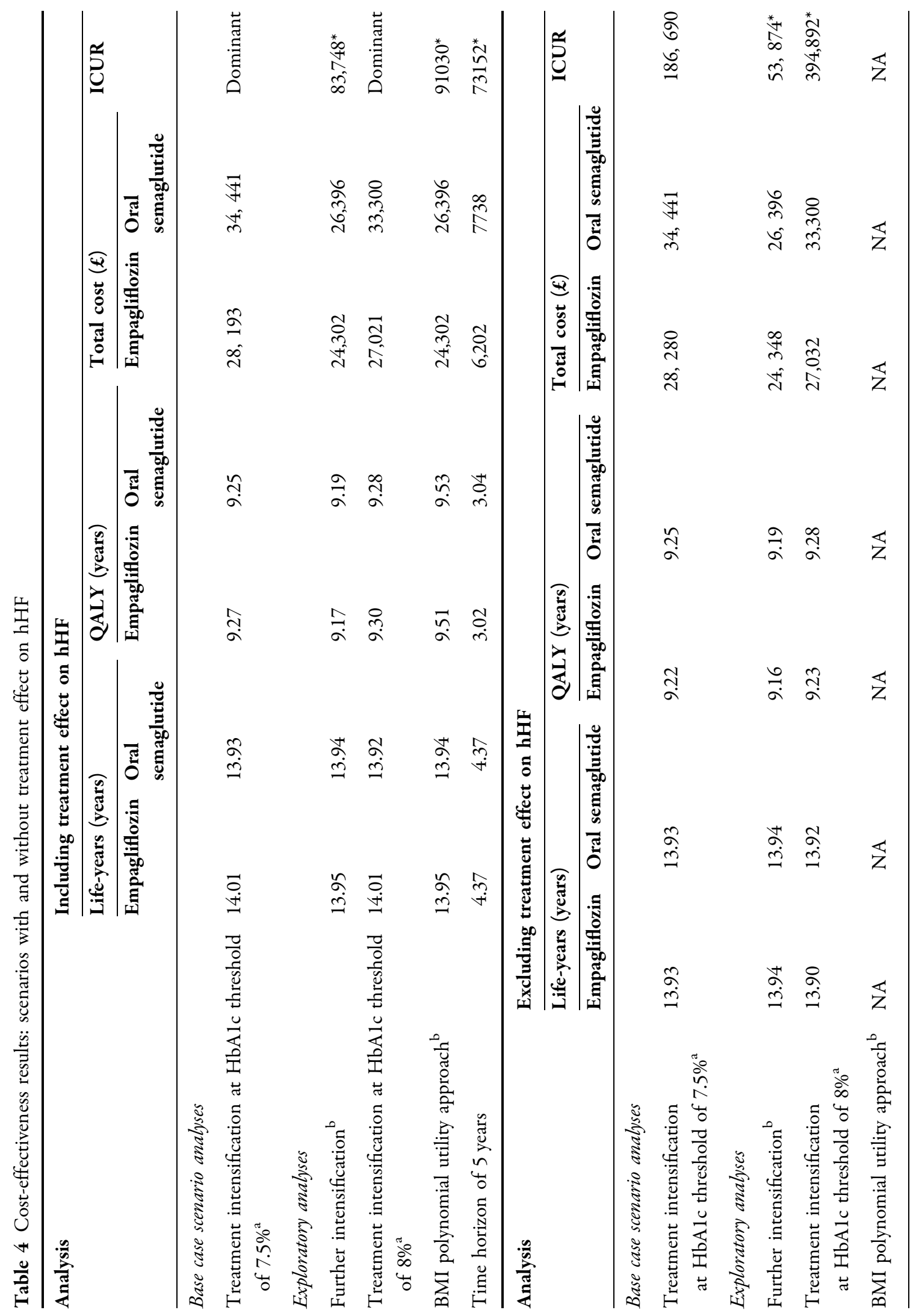




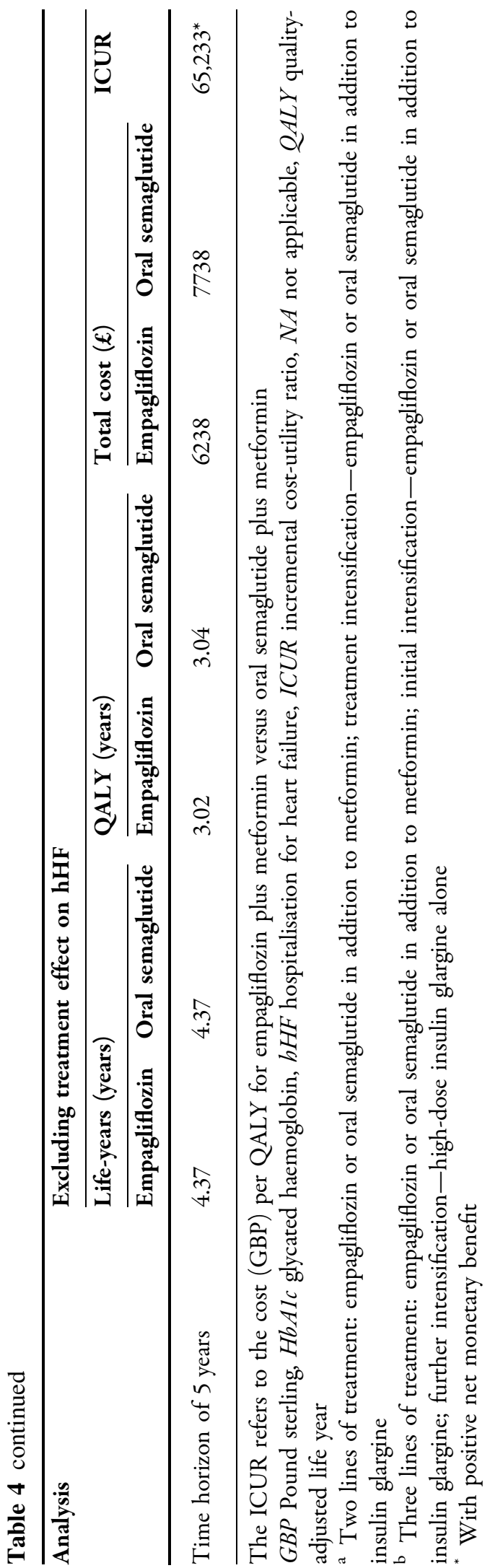

have used insulin as the treatment option for intensification [20,33-35], and this is accepted by health technology assessment agencies.

Also, the Institute for Clinical and Economic Review (ICER), an independent research institution in the US, estimated the cost-effectiveness of oral semaglutide versus several comparators including empagliflozin [36]. This study showed that, at the estimated net price, oral semaglutide is unlikely to meet cost-effectiveness thresholds compared with empagliflozin. The methodology followed by the ICER investigators deviated in many ways from the one applied in the current manuscript and from the one applied by Bain et al. Main differences are that they started from a US cohort and not the PIONEER 2 cohort and that they combined the treatment effects on risk factors measured in PIONEER 2 with direct treatment effects on CVD taken from PIONEER 6 combined with a network meta-analysis to obtain the effects of empaglifozin.

At the latest ADA conference, a poster was presented, based on PIONEER 2 but using two different cost-effectiveness models, on the costeffectiveness of oral semaglutide and empagliflozin [37]. As it was only a poster, comparing the methodologies is difficult. HbA1c of $8 \%$ was considered the moment to switch to insulin and stop oral semaglutide and empagliflozin (like Bain et al.) and analyses were run over 40 years. Here, the authors conclude that oral semaglutide is cost-effective.

In the above paragraphs, some of the limitations and how they were addressed were reported. Below, they are briefly repeated and some more are added. First, the UK cost of oral semaglutide is not yet plublicly available. We used the same cost as used by the manufacturer in their analyses. Second, the RR on hHF of empagliflozin versus GLP1 receptor agonists was used as no specific oral semagluide data are available. Hard outcomes were not included in PIONEER 2. Analyses without an impact on hHF have also been presented. Third, it is not known how long the therapy will be applied and what the next line therapy will be. A scenario analysis with three lines of therapy was added in which oral semaglutide and empagliflozin are stopped in third line, being after 4 to 5 years of therapy. 
Fourth, studies on empagliflozin have shown that it reduces the declines over time of eGFR and resulted in less end-stage renal disease [38]. So far, on oral semaglutide, based on published studies effects on albuminuria and ESRD are not shown. The occurrence of nephropathy in the model is driven by HbA1c reduction at the moment. As oral semaglutide results in a stronger reduction of $\mathrm{HbA} 1 \mathrm{c}$, this is a conservative approach for empagliflozin.

\section{CONCLUSIONS}

Driven by the strong difference in treatment costs, empagliflozin $25 \mathrm{mg}$ is a cost-effective treatment option versus oral semaglutide $14 \mathrm{mg}$, when used in addition to metformin, for the treatment of T2DM patients in the UK. Adding benefits on hospitalisation for heart failure empagliflozin becomes the dominant treatment.

\section{ACKNOWLEDGEMENTS}

The authors thank Andrew Ternouth for critical review of the manuscript and Manisha Panchal for supporting with data analysis.

Funding. This study and the Rapid Service Fee for publication were supported by funding from Boehringer Ingelheim, Ingelheim am Rhein, Germany.

Authorship. All named authors meet the International Committee of Medical Journal Editors (ICMJE) criteria for authorship of this article, take responsibility for the integrity of the work as a whole, and have given their approval for this version to be published.

Disclosures. Mafalda Ramos and Mark Lamotte are employees of IQVIA, which received consulting fees from Boehringer Ingelheim for their contribution to this work. Michael H. Cummings has received honoraria from Boehringer Ingelheim, Eli Lilly, Mylan, Napp, AstraZeneca, MSD and Amgen for expert advice on advisory boards and/or educational delivery. Anastasia Ustyugova is an employee of Boehringer Ingelheim International $\mathrm{GmbH}$. Syed I. Raza is an employee of Boehringer Ingelheim Ltd. Shamika U. de Silva is a former employee of Boehringer Ingelheim Ltd. Her current affiliation is Market Access, Biogen, Maidenhead, Berkshire SL6 4AY, UK.

Compliance with Ethics Guidelines. This article is based on previously conducted studies and does not contain any studies with human participants or animals performed by any of the authors.

Data Availability. All data generated or analysed during this study are included in this published article/as supplementary information files.

Open Access. This article is licensed under a Creative Commons Attribution-NonCommercial 4.0 International License, which permits any non-commercial use, sharing, adaptation, distribution and reproduction in any medium or format, as long as you give appropriate credit to the original author(s) and the source, provide a link to the Creative Commons licence, and indicate if changes were made. The images or other third party material in this article are included in the article's Creative Commons licence, unless indicated otherwise in a credit line to the material. If material is not included in the article's Creative Commons licence and your intended use is not permitted by statutory regulation or exceeds the permitted use, you will need to obtain permission directly from the copyright holder. To view a copy of this licence, visit http://creativecommons.org/licenses/by$\mathrm{nc} / 4.0 /$.

\section{REFERENCES}

1. Diabetes UK. The Cost of Diabetes Report (2014).

2. Cannon A, Handelsman Y, Heile M, Shannon M. Burden of illness in type 2 diabetes mellitus. J Manag Care Spec Pharm. 2018;24:S5-S13. 
3. Einarson TR, Acs A, Ludwig C, Panton UH. Prevalence of cardiovascular disease in type 2 diabetes: a systematic literature review of scientific evidence from across the world in 2007-2017. Cardiovasc Diabetol. 2018;17:83.

4. Juutilainen A, Lehto S, Ronnemaa T, Pyorala K, Laakso M. Type 2 diabetes as a "coronary heart disease equivalent": an 18-year prospective population-based study in Finnish subjects. Diabetes Care. 2005;28:2901-7.

5. Laing SP, et al. Mortality from heart disease in a cohort of 23,000 patients with insulin-treated diabetes. Diabetologia. 2003;46:760-5.

6. Baxter $M$, et al. Estimating the impact of better management of glycaemic control in adults with Type 1 and Type 2 diabetes on the number of clinical complications and the associated financial benefit. Diabet Med. 2016;33:1575-81.

7. Cefalu WT, et al. Cardiovascular outcomes trials in type 2 diabetes: where do we go from here? Reflections from a diabetes care editors' expert forum. Diabetes Care. 2018;41:14-311.

8. Rabizadeh S, Nakhjavani M, Esteghamati A. Cardiovascular and renal benefits of SGLT2 inhibitors: a narrative review. Int J Endocrinol Metab. 2019;17: e84353.

9. Wu JH, et al. Effects of sodium-glucose cotransporter- 2 inhibitors on cardiovascular events, death, and major safety outcomes in adults with type 2 diabetes: a systematic review and meta-analysis. Lancet Diabetes Endocrinol. 2016;4:411-9.

10. Zelniker TA, et al. SGLT2 inhibitors for primary and secondary prevention of cardiovascular and renal outcomes in type 2 diabetes: a systematic review and meta-analysis of cardiovascular outcome trials. Lancet. 2019;393:31-9.

11. Zhang XL, et al. Cardiovascular safety, long-term noncardiovascular safety, and efficacy of sodiumglucose cotransporter 2 inhibitors in patients with type 2 diabetes mellitus: a systemic review and meta-analysis with trial sequential analysis. J Am Heart Assoc. 2018;7(2):e007165. https://doi.org/10. 1161/JAHA.117.007165.

12. Tang $\mathrm{H}$, et al. Meta-analysis of effects of sodiumglucose cotransporter 2 inhibitors on cardiovascular outcomes and all-cause mortality among patients with type 2 diabetes mellitus. Am J Cardiol. 2016;118:1774-800.

13. Patorno E, et al. Empagliflozin and the risk of heart failure hospitalization in routine clinical care. Circulation. 2019;139:2822-30.
14. Fonseca VA. Defining and characterizing the progression of type 2 diabetes. Diabetes Care. 2009;32(Suppl 2):S151-156.

15. Ross SA. Breaking down patient and physician barriers to optimize glycemic control in type 2 diabetes. Am J Med. 2013;126:S38-48.

16. Reach G, Pechtner V, Gentilella R, Corcos A, Ceriello A. Clinical inertia and its impact on treatment intensification in people with type 2 diabetes mellitus. Diabetes Metab. 2017;43:501-11.

17. Buse JB, et al. 2019 Update to: management of hyperglycemia in type 2 diabetes, 2018. A consensus report by the American diabetes association (ADA) and the European association for the study of diabetes (EASD). Diabetes Care. 2020;43:487-93.

18. National Institute for Health and Care Excellence. Empagliflozin in combination therapy for treating type 2 diabetes. (2015).

19. National Institute for Health and Care Excellence. NICE guideline [NG28]: Type 2 diabetes in adults: management. (2019).

20. Bain SC, et al. Oral semaglutide versus empagliflozin, sitagliptin and liraglutide in the UK: longterm cost-effectiveness analyses based on the PIONEER clinical trial programme. Diabetes Ther. 2020;11:259-77.

21. Husain $\mathrm{M}$, et al. Oral semaglutide and cardiovascular outcomes in patients with type 2 diabetes. N Engl J Med. 2019;381:841-51.

22. Rodbard HW, et al. Oral semaglutide versus empagliflozin in patients with type 2 diabetes uncontrolled on metformin: the PIONEER 2 trial. Diabetes Care. 2019;42:2272-81.

23. Aroda VR et al. Oral semaglutide-the PIONEER Program Trials. in 2019 American Diabetes Association Annual Meeting (2019).

24. Palmer AJ, et al. The CORE Diabetes Model: projecting long-term clinical outcomes, costs and costeffectiveness of interventions in diabetes mellitus (types 1 and 2) to support clinical and reimbursement decision-making. Curr Med Res Opin. 2004;20(Suppl 1):S5-26.

25. Palmer AJ, et al. Validation of the CORE Diabetes Model against epidemiological and clinical studies. Curr Med Res Opin. 2004;20(Suppl 1):S27-40.

26. McEwan P, et al. Validation of the IMS CORE Diabetes Model. Value Health. 2014;17:714-24. 
27. National Institute for Health and Care Excellence. Guide to the methods of technology appraisal 2013; process and methods [PMG9]. (2013).

28. Kalra S, Kamaruddin NA, Visvanathan J, Santani R. Defining disease progression and drug durability in type 2 diabetes mellitus. Eur Endocrinol. 2019;15: 67-9.

29. Willis M, Asseburg C, Nilsson A, Johnsson K, Kartman B. Multivariate prediction equations for HbA1c lowering, weight change, and hypoglycemic events associated with insulin rescue medication in type 2 diabetes mellitus: informing economic modeling. Value Health. 2017;20:357-71.

30. National Institute for Health and Care Excellence. Carrying NICE over the threshold. (2015).

31. Soltoft F, Hammer M, Kragh N. The association of body mass index and health-related quality of life in the general population: data from the 2003 Health Survey of England. Qual Life Res. 2009;18: 1293-9.

32. National Institute for Health and Care Excellence. Algorithm for blood glucose lowering therapy in adults with type 2 diabetes. (2015).

33. Ericsson A, Lundqvist A. Cost effectiveness of Insulin Degludec Plus Liraglutide (IDegLira) in a fixed combination for uncontrolled type 2 diabetes mellitus in Sweden. Appl Health Econ Health Policy. 2017; 15:237-48.

34. Valentine WJ, Goodall G, Aagren M, Nielsen S, Palmer AJ, Erny-Albrecht K. Evaluating the cost-effectiveness of therapy conversion to insulin detemir in patients with type 2 diabetes in Germany: a modelling study of long-term clinical and cost outcomes. Adv Ther. 2008;25:567-84.

35. Brandle M, Azoulay M, Greiner RA. Cost-effectiveness and cost-utility of insulin glargine compared with NPH insulin based on a 10-year simulation of long-term complications with the Diabetes Mellitus Model in patients with type 2 diabetes in Switzerland. Int J Clin Pharmacol Ther. 2007;45:203-20.

36. Rind D, Guzauskas G, Fazioli K, Hansen R, Kumar V, Chapman R, Borrelli E, Bradt P, Pearson S. Oral Semaglutide for Type 2 Diabetes: Effectiveness and Value. Institute for Clinical and Economic Review, December 9, 2019. https://icer-review.org/material/ diabetes-final-evidence-report.

37. Liu AR, Bech PG, Fridhammar A, Nilsson A, Willis M, Nuhoho S. Cost effectiveness of oral semaglutide $14 \mathrm{mg}$ vs empagliflozin $25 \mathrm{mg}$ in Canada. Diabetes. 2020. https://doi.org/10.2337/db20-1163-P.

38. Zinman B, Wanner C, Lachin JM, Fitchett D, Bluhmki E, Hantel S, et al. Empagliflozin, cardiovascular outcomes, and mortality in type 2 diabetes. N Engl J Med. 2015;373(22):2117-288. 